Methods 27 healthy volunteers were randomly assigned to a vaccinated $(n=14)$ or a control $(n=13)$ group for Ty21a typhoid vaccine. Peripheral blood was collected from all volunteers prior to vaccination and 18 days following immunisation or recruitment. Mucosal samples (15 jumbo biopsies from duodenum $(n=25) \pm$ colon $(n=18)$ ) were collected from all volunteers at gastroscopy $+/-$ sigmoidoscopy on day 18. Mononuclear cells were isolated from mucosal tissue by disruption and collagenase digestion, and from blood by differential centrifugation. Cells were stimulated with Ty21a or control antigens, and stained for surface phenotype and intracellular cytokine production. Antigen-specific IFN- $\gamma$, TNF- $\alpha$, and IL-2 production was determined by flow cytometric analysis for $\mathrm{CD}^{+} / \mathrm{CD}^{+}$and $\mathrm{CD}^{+} / \mathrm{CD}^{-}\left(\mathrm{CD}^{+}\right)$lymphocytes. Humoural $\operatorname{IgA}, \operatorname{IgM}$ and $\operatorname{IgG}$ responses in blood were examined in relation to mucosal and peripheral cellular responses.

Results Oral immunisation with Ty21a significantly increased the proportion of antigen-specific cytokine-producing CD8-positive $(p<0.05)$ and CD8-negative $(p<0.05)$ lymphocytes within the duodenal mucosa, but no specific response was seen in colon. CD8negative lymphocytes within the duodenal mucosa adopted a significantly more poly-functional phenotype following vaccination, expressing 2 or 3 cytokines simultaneously, while in contrast antigen-specific cytokine-producing CD8-positive lymphocytes in the duodenal mucosa were mono-functional expressing a single cytokine. In blood, the proportion of antigen-specific cytokineproducing CD8-positive lymphocytes was increased $(p<0.05)$ following oral vaccination, but there was no significant increase in cytokine-producing CD4-positive lymphocytes. Differences in functionality of antigen-specific cytokine responses were less marked in peripheral blood lymphocytes following vaccination.

Conclusion These data show an antigen-specific response in human gut mucosal lymphocytes following oral vaccination, and directly demonstrate different immune functionality of CD8-positive compared to CD8-negative mucosal lymphocytes. These responses were more informative than surrogate measurements in peripheral blood lymphocytes. The absence of a detectable cognate response from the colon may indicate compartmentalisation of the gut mucosal response to the embryological mid-gut, where typhoid antigen is likely presented at immune inductive sites.

Competing interests None declared.

\section{PWE-131 PROPOFOL DEEP SEDATION FOR SMALL BOWEL ENTEROSCOPY IN ELDERLY PATIENTS IN A WORLD GASTROENTEROLOGY ORGANISING ENDOSCOPY TRAINING CENTER IN THAILAND}

doi:10.1136/gutjnl-2012-302514d.131

S Amornyotin,* S Kongphlay. Department of Anesthesiology, Faculty of Medicine Siriraj Hospital, Mahidol University, Bangkok, Thailand

Introduction The aim of the study is to compare and evaluate the clinical efficacy of propofol deep sedation (PDS) for small bowel enteroscopy (SBE) procedure in elderly patients in a teaching hospital in Thailand.

Methods This study was a retrospective study. All SBE patients were classified into two groups by using age: group 1 (Age $<65$ years) and group GA (Age $\geq 65$ years). The primary outcome variable of the study was the successful completion of the procedure. Failed procedure is defined as the procedure can not be completed by using PDS technique or sedation-related serious adverse events such as severe hypoxaemia, severe cardiorespiratory instability, are occurred. The secondary outcome variables were sedation-related complications, haemodynamic parameters and mortality rate.

Results 116 patients underwent SBE procedures by using PDS technique. Premedications were none before the procedure. After matching gender, weight, ASA physical status and indications of procedures, there were 45 patients in group 1 and 28 patients in group 2. There were no significant differences in gender, weight, height, ASA physical status and indications of procedures among the two groups. All procedures were successful completion but one in group 1. Mean dose of propofol, fentanyl and midazolam in both groups was comparable. There were no significant differences in the complication rate, mortality rate and haemodynamic parameters between the two groups.

Conclusion In the setting of developing country, PDS for SBE procedure in elderly patients by experienced anaesthesiologist with appropriate monitoring were relatively safe and effective. Sedationrelated complications in elderly patients are relatively higher than in the younger patients, but not significantly different.

Competing interests None declared.

\section{PWE-132 ENHANCED EXPRESSION OF SECRETORY PHOSPHOLIPASE A2 AND CRYPTDINS IN SMALL INTESTINAL PANETH CELLS FOLLOWING TRICHINELLA SPIRALIS INFECTION}

doi:10.1136/gutjnl-2012-302514d.132

W S Wan Shahida, ${ }^{*}$ R J Morgan-Walsh, Y R Mahida. Institute of Infection, Immunity \& Inflammation, University of Nottingham, Nottingham, UK

Introduction $T$ spiralis infection leads to a $T$ cell-dependent enteropathy characterised by villus atrophy, crypt hyperplasia and an increase in Paneth and goblet cells. Paneth cells express a number of antimicrobial peptides and proteins. Our aim was to investigate changes in the expression of cationic antimicrobial peptides and proteins that are normally expressed by Paneth cells.

Methods Small intestinal epithelial cells were isolated from control mice and those infected with Tspiralis. Concentrated cell extracts (in acetic acid) were studied by acid urea-polyacrylamide gel electrophoresis (AU-PAGE) and Western blot analysis. Samples with similar protein concentrations were used to assess antimicrobial activity against Escherichia coli, after $5 \mathrm{~h}$ of incubation at $37^{\circ} \mathrm{C}$, using the following equation: antimicrobial activity $=[(\mathrm{OD} 620$ of control solution-OD620 of sample)/OD620 of control solution] $\times 100$.

Results The establishment of infection with the nematode was confirmed by the presence of worms in the small intestinal lumen, changes in mucosal architecture and increase in Paneth and goblet cell numbers. In contrast to controls, AU-PAGE analysis of Paneth cell-containing small intestinal epithelial cell extracts from Tspiralisinfected mice showed two prominent bands. AU-PAGE-Western blot and amino acid sequence analyses identified one of these bands to be secretory phospholipase A2. Sequences for cryptdins were detected in the second prominent band. Acid extracts of epithelial cells isolated from $T$ spiralis-infected mice showed significantly greater antimicrobial activity, compared to those from control mice [mean 54.7 (SEM 8.7)\% vs 7.3 (3.5)\%; $\mathrm{p}=0.001$ ].

Conclusion Following $T$ spiralis infection, there was an increase in small intestinal epithelial expression of secretory phospholipase A2 and cryptdins. Enhanced production of these Paneth cell-derived peptides is likely to mediate greater antimicrobial activity against luminal bacteria in Tspiralis-infected small intestine.

Competing interests None declared.

\section{Hepatobiliary II \\ PWE-133 INCREASED LEVELS OF NEUTROPHIL GELATINASE ASSOCIATED LIPOCALIN (NGAL) IN THE PLASMA OF CHOLANGIOCARCINOMA PATIENTS}

doi:10.1136/gutjnl-2012-302514d.133

${ }^{1,2} \mathrm{C}$ A Wadsworth, ${ }^{*} 1,2,3 \mathrm{~V}$ M Horneffer-van der Sluis, ${ }^{1,2} \mathrm{~A}$ Zabron, ${ }^{4} \mathrm{~K}$ M Boberg, ${ }^{5} \mathrm{~S} P$ Pereira, ${ }^{3} \mathrm{R}$ J Edwards, ${ }^{1,2} \mathrm{~S}$ D Taylor-Robinson, ${ }^{1,2} \mathrm{~S}$ A Khan. ${ }^{1}$ Department of 